\title{
PRATIQUES DE MÉDIATION INFORMATIONNELLE SUR FACEBOOK : L'APPROPRIATION POLITIQUE DE LA PRESSE QUOTIDIENNE RÉGIONALE LORS DE LA CAMPAGNE PRÉSIDENTIELLE DE 2017
}

\author{
Franck Bousquet, Julien Figeac, Guillaume Cabanac, Camille Noûs
}

E.N.S. Editions | « Mots. Les langages du politique »

$2020 / 2 \mathrm{n}^{\circ} 123$ | pages 81 à 102

ISSN 0243-6450

ISBN 9791036202490

Article disponible en ligne à l'adresse :

https://www.cairn.info/revue-mots-2020-2-page-81.htm

Distribution électronique Cairn.info pour E.N.S. Editions.

(C) E.N.S. Editions. Tous droits réservés pour tous pays.

La reproduction ou représentation de cet article, notamment par photocopie, n'est autorisée que dans les limites des conditions générales d'utilisation du site ou, le cas échéant, des conditions générales de la licence souscrite par votre établissement. Toute autre reproduction ou représentation, en tout ou partie, sous quelque forme et de quelque manière que ce soit, est interdite sauf accord préalable et écrit de l'éditeur, en dehors des cas prévus par la législation en vigueur en France. Il est précisé que son stockage dans une base de données est également interdit. 
De la racine à l'extrémisme : discours des radicalités politiques et sociales

\section{Pratiques de médiation informationnelle sur Facebook : l'appropriation politique de la presse quotidienne régionale lors de la campagne présidentielle de 2017}

Information-sharing practices on Facebook: the political appropriation of the daily press during the 2017 French presidential campaign

Prácticas de mediación informacional en Facebook: la apropriación política de la prensa cotidiana regional durante la campaña presidencial de 2017

Franck Bousquet, Julien Figeac, Guillaume Cabanac et Camille Noûs

\section{(2) OpenEdition}

\section{Édition électronique}

URL : http://journals.openedition.org/mots/26797

DOI : $10.4000 /$ mots. 26797

ISBN : 979-10-362-0250-6

ISSN : 1960-6001

Éditeur

ENS Éditions

Édition imprimée

Date de publication : 10 septembre 2020

Pagination : 81-102

ISBN : 979-10-362-0249-0

ISSN : 0243-6450

Distribution électronique Cairn

\section{CAIRN INEO}

CHERCHER, REPÉRER, AVANCER.

Référence électronique

Franck Bousquet, Julien Figeac, Guillaume Cabanac et Camille Noûs, « Pratiques de médiation informationnelle sur Facebook : l'appropriation politique de la presse quotidienne régionale lors de la campagne présidentielle de 2017 », Mots. Les langages du politique [En ligne], 123 | 2020, mis en ligne le 02 janvier 2024, consulté le 03 juillet 2020. URL : http://journals.openedition.org/mots/26797 ; DOI : https://doi.org/10.4000/mots.26797 


\section{Pratiques de médiation informationnelle sur Facebook : l'appropriation politique de la presse quotidienne régionale lors de la campagne présidentielle de $2017^{*}$}

Les médias d'information sont historiquement liés au fonctionnement de la vie politique. Principaux pourvoyeurs d'informations alimentant le débat public et permettant aux citoyens de faire un choix électoral éclairé, ils sont l'un des piliers de la théorie démocratique traditionnelle (Bougnoux, 2006). Dans leurs éditoriaux ou leurs prises de position publiques, les journalistes ne manquent jamais de revendiquer ce rôle à l'origine de leur reconnaissance sociale.

Cependant, depuis le début des années 2000 , les pratiques informationnelles sont en profonde mutation. En quelques décennies, de nouveaux acteurs ne produisant pas directement d'informations et d'autres en marge du monde journalistique contestent le monopole de l'information politique des médias classiques (Marty et al., 2012). La presse d'information générale a été la principale victime de ce mouvement. Maîtresse de l'agenda jusqu'au début des années 2000, elle traverse depuis une crise économique majeure qui se double d'une défiance grandissante des populations à son égard.

Les pratiques informationnelles ont effectivement profondément changé (Jouët, Rieffel éd., 2013) et désormais la médiation de l'information est en partie entre les mains d'intermédiaires de différentes natures, qui sont qualifiés d'infomédiaires (Rebillard, Smyrnaios, 2010). La question de la médiation est par conséquent devenue fondamentale, car les médias eux-mêmes sont obligés d'en référer à ces nouveaux acteurs pour atteindre leurs publics.

* $\quad$ Recherche réalisée dans le cadre du projet LisTIC (projet ANR-16-CE26-0014-01) financé par l’Agence nationale de la recherche.

Université Toulouse 3, LERASS

franck.bousquet@univ-tlse3.fr

Université Toulouse 2, LISST

julien.figeac@univ-tlse2.fr

Université Toulouse 3, IRIT

guillaume.cabanac@univ-tlse3.fr

Laboratoire Cogitamus / Cogitamus Laboratory

camille.nous@noussommesluniversite.fr

Mots. Les langages du politique $\mathrm{n}^{\circ} 123$ juillet $2020 \bullet 81$ 
Parmi ces intermédiaires informationnels, les réseaux socionumériques (RSN) sont devenus centraux. Outils mêlant information, communication et exposition de soi, ils se sont rendus incontournables pour l'audience de presque tous les médias d'information (Chadwick, 2013; Jenkins, 2008). Tous les titres de presse cherchent désormais à développer leur présence au sein des pratiques d'échanges d'informations des utilisateurs de ces réseaux (Le Caroff, 2018). Ils sont cependant en concurrence avec de nombreux autres acteurs non journalistiques produisant aussi de l'information que l'on peut qualifier de politique, que ce soit à des fins partisanes, diplomatiques, commerciales ou humoristiques.

Dans le même temps, la circulation de l'information sur les RSN se fait au sein de regroupements de personnes partageant des centres d'intérêt communs. Ces groupes d'utilisateurs, que nous qualifions de communautés, choisissent, diffusent, mettent en commun et commentent des informations qui semblent en accord avec ce qui les rassemble. Ainsi, les groupes politiques ont tous des comptes ou des pages sur les RSN, à l'intérieur desquels ils sélectionnent des informations qui servent leurs intérêts partisans, mobilisent leurs supporters ou nourrissent, voire attisent, des débats ou des arguments qui leur semblent importants. Nous assimilons ici ces réseaux d'utilisateurs à des "communautés politiques». Cette expression renvoie aux collectifs d'utilisateurs qui ont contribué à un groupe ou une page Facebook explicitement affiliés à un mouvement politique. Ces individus forment une communauté dans la mesure où ces internautes doivent le plus souvent avoir été autorisés à accéder au contenu de ces pages après en avoir formulé la demande auprès des administrateurs. Par cette demande d'adhésion, puis sa validation, ils signalent un intérêt commun pour un même mouvement politique. Nous considérons alors qu'ils se rallient à une même communauté politique s'ils ont partagé un contenu dans au moins une des pages Facebook affiliées à un même candidat ou à un même parti.

La médiation informationnelle est au centre des pratiques de ces communautés politiques qui transmettent, commentent, jugent, voire produisent des nouvelles qui leur semblent importantes à partager. Dans un contexte où l'on parle de plus en plus de bulles de filtres, de polarisation idéologique des opinions, de mal-information ou de fake news, révéler les pratiques réelles de médiation informationnelle, constatées par des observations empiriques de groupes politiquement engagés sur les RSN, représente donc un enjeu de toute première importance.

C'est à cet enjeu que cette étude est vouée. Mais, face à la diversité des sources d'informations qui circulent dans ces communautés, nous voudrions nous concentrer sur un certain type de presse qui passe souvent sous les radars des questionnements scientifiques ou politiques malgré son importance sociale et sa place dans les pratiques médiatiques contemporaines. Les 
médias locaux et régionaux, et en particulier la presse, sont en effet des sources informationnelles majeures dans la vie quotidienne des Français, comme l'attestent les chiffres de diffusion de ces titres et l'audience de leurs sites internet (Bousquet, 2015). Cependant, peu de recherches étudient leurs usages, leurs contenus et la façon dont les informations qu'ils produisent circulent et sont commentées dans les RSN. Cet article propose de pallier ce manque en questionnant plus précisément les pratiques de médiation informationnelle des principales communautés politiques françaises contemporaines avant d'analyser en détail leur utilisation de la presse locale.

Notre corpus d'étude est constitué des pages Facebook nationales et régionales des cinq principaux partis et mouvements politiques en lice lors de la campagne présidentielle de 2017 (FN, LFI, LR, PS et LREM). La forte activité de ces pages lors de cette période ainsi que leur concentration sur ce qui constitue le cœur de leur positionnement politique nous semble en effet propice à l'observation de leur utilisation des sources informationnelles multiples, que ce soient des articles tirés de la presse, des sites des partis politiques, des blogs de militants, d'instituts de sondages, etc. Pour répondre à notre questionnement et mettre en lumière la place de la presse quotidienne régionale (PQR) dans les pratiques de médiation informationnelle de ces communautés politiques, nous aborderons successivement plusieurs points.

Tout d'abord, nous examinerons la place qu'occupent aujourd'hui les médias centraux (Institut Montaigne, 2019) au sein de l'ensemble des sources d'information mobilisées par nos communautés politiques pourvoir si la pluralité des informations favorisées par ces plateformes numériques concurrence, voire défie, la légitimité des discours journalistiques. Puis nous mettrons en lumière la place de la presse locale et régionale au sein de ces pratiques de médiation. Dans un second temps, nous analyserons le recours à ce type de presse et les usages qui en sont faits en soulignant les différences constatées entre les communautés politiques. Nous formulons effectivement l'hypothèse que la singularité des informations locales, notamment la place qu'occupent les faits divers au sein des colonnes de la PQR (Berthaut, Darras, Laurens, 2009), est probablement plus en phase avec les préoccupations politiques et l'ancrage territorial de certains mouvements. Finalement, cette étude apportera des éléments pour comprendre les usages que les acteurs politiques font des informations produites par la presse locale et régionale afin de mieux analyser les implications politiques de certains choix éditoriaux, relatifs par exemple à la place accordée aux informations de quartier et aux faits divers. Mais avant de présenter la méthode et les résultats de cette recherche, il convient de motiver notre démarche en justifiant pourquoi il est aujourd'hui pertinent de questionner la place de la $P Q R$ dans les pratiques de médiation des communautés politiques sur Facebook. 


\section{La PQR dans les pratiques de médiation informationnelle sur Facebook}

Apparue en même temps que la presse nationale au début du Xvıı siècle, la presse locale est avant tout un média capable de réunir une communauté de lecteurs se reconnaissant dans le territoire qu'il dessine. C'est aussi un média de service qui donne une place importante à des informations pratiques, éditorialisées uniquement à la marge et qui sont utiles à la vie quotidienne de ses lecteurs. Différent dans tous les pays du monde du fait d'histoires politiques, sociales et économiques diverses, le rapport au territoire et au public est cependant une constante dans la réalité médiatique locale. Des études très récentes continuent à démontrer que, pour un individu, la lecture d'un média local renvoie à son intégration à une communauté territoriale (Hayes, Lawless, 2018). Elle est d'ailleurs très liée à la participation politique : ceux qui votent le plus sont aussi ceux qui consomment régulièrement un média local.

Au fil des années, les évolutions socio-économiques (concentration à travers quelques grands groupes et concurrence généralisée sur internet), sociopolitiques (affaiblissement des attaches politiques individuelles et épuisement du système partisan traditionnel) et sociotechniques (développement d'une offre en ligne fractionnée et consommation des nouvelles sur des supports numériques) ont profondément mis à mal le système médiatique local et régional dans l'ensemble de l'Europe occidentale et particulièrement en France. Malgré cela, la $P Q R$ qui s'était affirmée comme un acteur majeur de la redéfinition du rapport politique, culturel et économique au local, du début de la Troisième République jusqu'aux années 1960, continue de dominer le paysage.

Les titres de la PQR ont en effet construit des monopoles sur des territoires de plus en plus étendus grâce à des politiques de rachat et d'entente (Le Floch, 1997). Ainsi, sur de nombreux territoires, un seul titre de PQR subsiste et se trouve être le seul acteur capable d'apporter une information détaillée à un niveau de granularité très fin : celui de la commune ou du quartier. Cette évolution a eu deux conséquences. Tout d'abord, la PQR est demeurée un acteur majeur de l'information locale qui n'a pas vraiment de concurrence et qui, malgré une perte de lectorat et de recettes publicitaires, conserve une importance sociale majeure. Ensuite, les titres de PQR, du fait de leur situation de monopole, se sont progressivement retirés de l'arène politique partisane pour construire une information censée s'adresser à tous les lecteurs d'un territoire, quelles que soient leurs convictions politiques (Quéré, Dulong, 1978). Renforçant leur offre d'information service (Bousquet, 2015), ils ont aussi développé des propositions éditoriales co-construites avec les principaux pouvoirs du territoire et donné une importance grandissante aux faits divers (Frisque, 2010). Presse populaire par définition, la PQR a toujours accordé une place de choix 
aux faits divers locaux, événements qu'elle est la seule à pouvoir, voire à vouloir, traiter. Mais cette place était associée à une éditorialisation politique du titre qui conférait à l'actualité politique locale un sens articulé aux débats nationaux. Aujourd'hui, les faits divers sont à la fois le signe de la volonté des titres de s'adresser à tous les publics, quelle que soit leur coloration politique, et d'adopter une stratégie agressive de conquête des publics construite sur le sensationnalisme. À tel point que certains parlent de McJournalism pour qualifier la fabrication de l'information locale (Franklin, 2005).

Au vu de cette situation, il est intéressant à plus d'un titre de chercher à étudier la place de la $P Q R$ dans les pratiques de médiation informationnelle sur Facebook. Il apparaît notamment que Facebook, comme Google d'ailleurs, a décidé depuis le début de l'année 2019 de donner une place particulière à l'information de proximité. Dispositif de sociabilité fondé sur la mise en relation de personnes, le partage de contenus et la constitution de groupes autour d'intérêts communs, Facebook représente en effet un vecteur particulièrement intéressant pour les titres de presse locale. Il a la capacité de fédérer des individus ou des communautés partageant des intérêts similaires sur des territoires précis et ainsi de construire un lectorat aux caractéristiques bien identifiées et géographiquement localisé. Il mêle la communication interpersonnelle et la communication médiatisée à un niveau d'intégration jamais atteint (Cardon, 2012 ; Mercier et al., 2018), proposant ainsi la réalisation de l'un des objectifs initiaux de la presse locale. Cependant la nature des liens tissés et des communautés constituées pose question. Comme le souligne Dominique Boullier, «[c]e sont elles (ces plateformes) qui recomposent les affinités dans leur maillage territorial, reterritorialisé, qui constitue l'enveloppe globale dont on déplore souvent la perte du point de vue de l'espace public» (Boullier, 2014, p. 105). La question du rôle de l'information de proximité ainsi que de sa nature se trouve ainsi reposée à l'aune des usages sociaux de ces plateformes - et de Facebook en particulier - dans la constitution des réseaux de sociabilité.

Ce type de résultat peut ainsi nous renseigner sur deux aspects : le premier concerne la perception de l'offre éditoriale par les lecteurs et le second une certaine réalité sociale des choix éditoriaux de la $P Q R$ (qui ne se révèlerait que par l'intermédiaire des usages sociaux de l'information construits par certains types de lecteurs).

\section{Présentation de la méthode et du corpus}

\section{Délimitation du périmètre du corpus de pages Facebook}

Notre recherche analyse comment les principaux mouvements politiques français et leurs sympathisants, voire militants, ont utilisé Facebook durant la 
campagne de l'élection présidentielle de 2017 pour échanger des informations et alimenter leurs débats. Cette analyse est fondée sur des traces d'usage, collectées entre octobre 2016 et mai 2017 sur les pages Facebook des principales communautés politiques françaises.

Comme la collecte des données des pages Facebook est complexe, nous avons restreint notre corpus aux contenus promouvant les cinq principaux candidats : celles soutenant les candidatures de Jean-Luc Mélenchon (La France insoumise, LFI), Benoît Hamon (Parti socialiste, PS), François Fillon (Les Républicains, LR), Marine Le Pen, la dirigeante du Front national ${ }^{1}$ (FN), et Emmanuel Macron, le fondateur du parti La République En Marche (LREM), qui a remporté cette élection.

Nous avons considéré ce corpus comme représentatif car il regroupe 252 pages publiques Facebook, soit une cinquantaine de pages par communauté politique, afin d'inclure leurs principales pages officielles aux niveaux national, régional, départemental et des grandes agglomérations françaises.

\section{Méthode d'analyse des sources d'information partagées par les communautés politiques}

Pour analyser finement les usages de ces différentes communautés politiques, il s'est révélé pertinent de collecter et d'anonymiser les contenus de ces pages Facebook à l'aide de Netvizz (Rieder, 2013). Ce logiciel extrait une grande diversité de données, dont le nombre et le contenu des commentaires, le nombre et le type de contenus partagés, le nombre de «J'aime», etc. Ces données permettent de quantifier les types de publications partagées en fonction du contenu médiatisé (du texte, des photos, des vidéos, etc.). II apparaît alors, comme le montre la figure 1 , que presque un tiers des publications partagées, tous partis confondus, contiennent un lien hypertexte pointant vers un article de presse ou vers une source d'information extérieure à Facebook (31\%) - le présent article traite exclusivement de ces publications.

Ce corpus d'hyperliens regroupe 8838 sources d'information distinctes, caractérisées par leur domaine internet tel que lemonde.fr (voir tableau 1), qui ont été partagées par les communautés politiques au sein de leurs pages Facebook. Nous avons ensuite cherché à caractériser et coder manuellement ces différents types de sources d'information en distinguant : les sites de la presse écrite (nationale, régionale, magazine), les sites des chaînes de télévision ou de radio, les sites des partis politiques, des ONG, des associations, des instituts de sondage, etc. Comme plusieurs articles ont pu être partagés depuis la même source, notamment depuis les principaux quotidiens nationaux, ce corpus contient un grand nombre d'articles $(n=355503)$ partagés depuis un

1. Le Front national a été rebaptisé Rassemblement national le 1er juin 2018. 


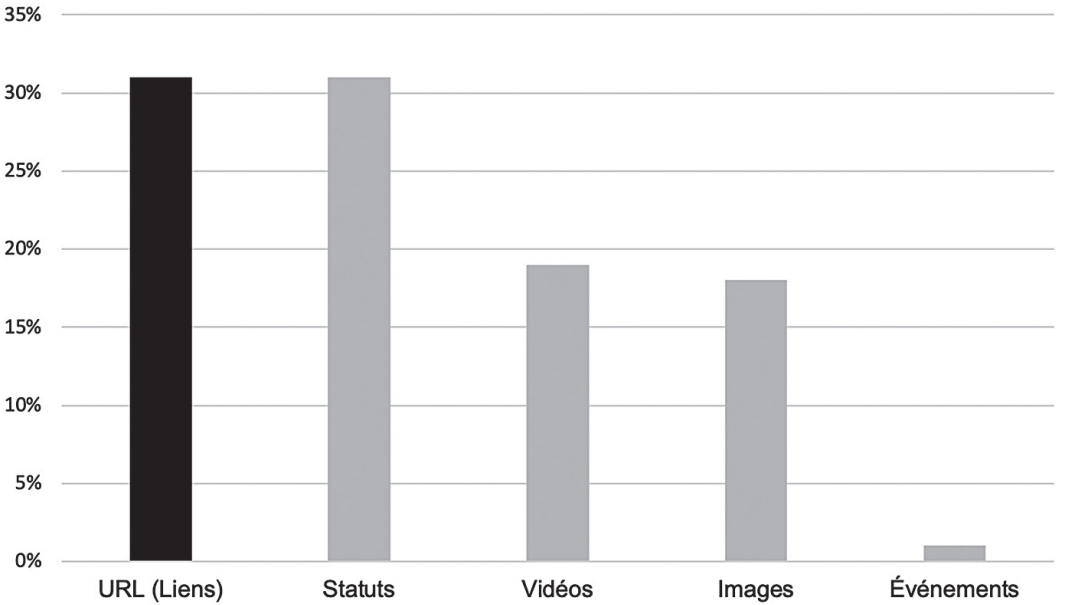

Figure 1. Part des types de publications Facebook échangées entre octobre 2016 et mai 2017

même domaine internet $(n=8838)$. Parmi ces sources d'information, nous avons réussi à identifier leur origine dans 95,04 \% des cas (tableau 1). Dans le cadre de cet article, nous ferons un focus sur les sources d'information qui renvoient à la presse écrite et, plus précisément, à la presse écrite régionale.

\begin{tabular}{l|c|c}
\hline Occurrence des sources & Part des sources & Part des occurrences \\
\hline 1 ou 2 & $62,39 \%$ & $1,95 \%$ \\
\hline 2 à 10 & $24,08 \%$ & $3,01 \%$ \\
\hline Plus de 10 & $13,53 \%$ & $95,04 \%$ \\
\hline Total (Taille de l'échantillon) & $100,00 \%(8838)$ & $100,00 \%(355503)$ \\
\hline
\end{tabular}

Tableau 1. Les taux de catégorisation des sources citées dans les publications

\section{Méthode d'analyse des contenus lexicaux des publications}

Nous avons analysé les contenus lexicaux des publications diffusées entre octobre 2016 et mai 2017 par les communautés politiques. Ce corpus textuel permet d'analyser les sujets et les thèmes qui ont été partagés au sein de cette arène numérique en identifiant notamment la place qu'ils occupent parmi les messages publiés par les différents réseaux d'acteurs que nous avons identifiés. Pour analyser ce corpus, nous avons utilisé la méthode Reinert (Reinert, 1990) implémentée dans le logiciel libre Iramuteq (Ratinaud, 2014). Elle permet de déterminer les thématiques qui structurent un corpus, sur la base d'une 
classification hiérarchique descendante qui peut être décrite comme une succession de bipartitions reposant sur une analyse factorielle des correspondances. Les «mondes lexicaux» (Reinert, 1990) qui se dégagent présentent alors les différentes thématiques abordées dans le corpus. L'analyse que nous allons présenter maintenant examine le contenu de ces différentes classes thématiques afin de rendre compte des principaux thèmes.

\section{Résultats}

Une surreprésentation de la $P Q R$ dans les pratiques de médiation informationnelle du FN

Tout d'abord, que révèle notre corpus des pratiques d'information et de médiation informationnelle mises en place dans Facebook par les principaux groupes politiques lors de la campagne présidentielle de 2017 ? Le premier résultat de notre étude indique que si la production des médias sociaux (bavardage, échanges d'idées, partage de photos et vidéos) a occupé la première place dans les pratiques de médiation informationnelle de toutes les communautés politiques engagées dans le débat électoral, la place de la presse traditionnelle (c.-à-d. les posts mentionnant un lien vers un article publié dans un titre de la presse) n'est pas négligeable pour autant (voir figure 2). Pour toutes les communautés étudiées, le recours aux contenus produits par la presse traditionnelle se situe entre $20 \%$ et $30 \%$ de ce qui est diffusé. En outre, ce ne sont pas les groupements politiques liés aux partis situés aux extrémités du spectre politique (ici LFI et le FN) qui ont le plus recours aux contenus des médias sociaux. Au contraire, les groupements issus de ces deux partis sont ceux qui ont le moins diffusé ce type de contenu ( $35 \%$ pour LFI et $43 \%$ pour le $\mathrm{FN}$ ) alors que pour LREM ce même chiffre est de $53 \%$.

Notons enfin que LFI est le groupement qui a le plus recouru à des contenus produits par les instances du parti, tels que les transcriptions de discours et les communiqués de presse (19\%). Ce résultat témoigne de l'importance de la centralisation partisane pour ce parti. On observe également que les pages du FN ont véhiculé beaucoup d'informations publiées par des sites militants $(11 \%)$ et des pages personnelles ( $12 \%)$. Ce fait renvoie à la forte production et circulation de contenus politiques par des individus ou des groupements d'extrême droite en marge de l'appareil du FN qui viennent souvent éclairer, compléter et accentuer un discours officiel plus régulé et euphémisé depuis que ce parti tente d'en gommer les aspects les plus violents.

Intéressons-nous désormais aux contenus englobés dans la catégorie "Presse» afin d'examiner quels sont les médias les plus utilisés en fonction de chaque groupement politique. Là encore, nous allons nous concentrer sur 
$60 \%$

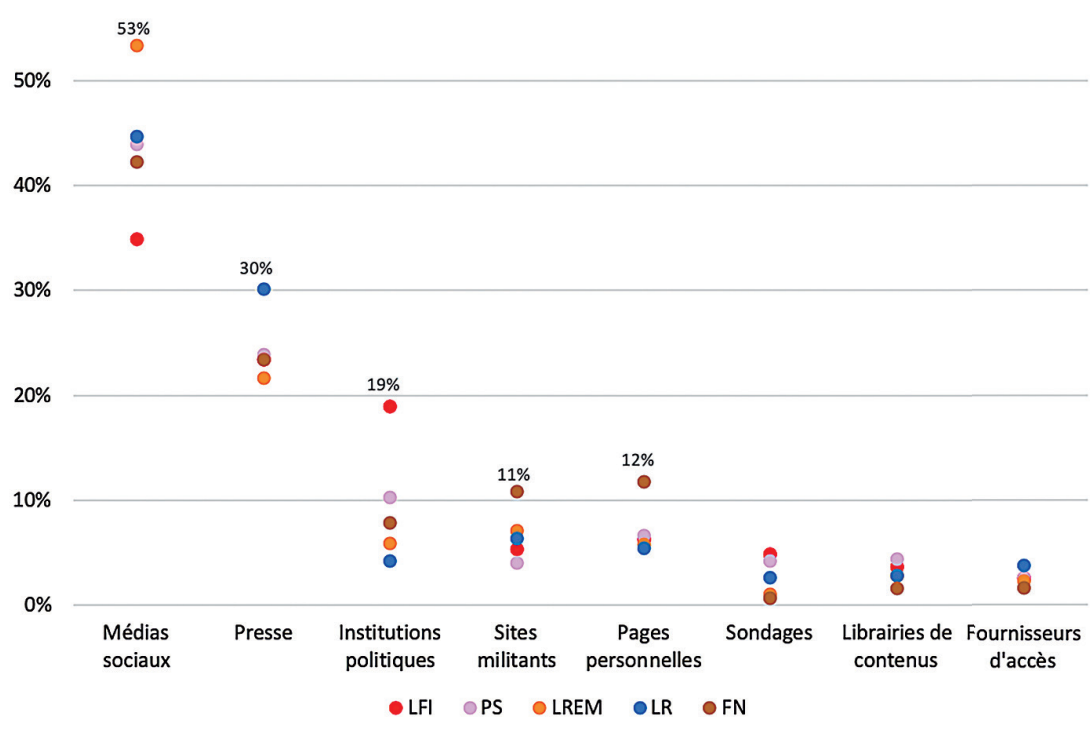

Figure 2. Part des types de contenus dans les publications Facebook $(n=355503)$

la place des médias locaux et de la PQR. La figure 3 révèle trois enseignements. Premièrement, parmi les sources médiatiques journalistiques, la presse quotidienne nationale (PQN) occupe toujours un rôle fondamental pour les groupements à caractère politique. Presse politique par excellence, la PQN a toujours représenté la presse de la nation dans le système médiatique français, celle qui participe à la construction politique du pays et qui est responsable de son animation. Il n'est donc pas étonnant de la trouver dans cette position dans le cadre de groupements politiques participant à une élection nationale.

Deuxièmement, et c'est un résultat contre-intuitif, les médias audiovisuels nationaux ne représentent qu'environ $20 \%$ des contenus de presse partagés par les différents groupements politiques. Alors que l'information audiovisuelle arrive régulièrement en tête des moyens d'information des Français, elle occupe une place très limitée ici. Il se peut que les administrateurs de communautés ne considèrent pas ces médias comme des sources d'information écrites légitimes à partir desquelles relayer des articles. Ils préfèrent apparemment se tourner vers les titres dont l'écrit est le cœur de métier.

Troisièmement, et c'est un résultat très significatif pour nos interrogations, la place de la presse locale est significativement différente selon les groupements politiques : elle est trois fois plus importante dans les pratiques de médiation informationnelle du groupe FN. Avec plus de $18 \%$ des informations 
médiatiques diffusées qui relèvent de la $P Q R$, le groupement FN est celui qui utilise le plus la PQR et aussi celui qui utilise le moins les sources audiovisuelles, la presse magazine et les médias natifs du web. Ce résultat, très significatif, doit être interrogé.

$60 \%$

$50 \%$

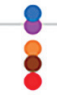

$40 \%$

$30 \%$

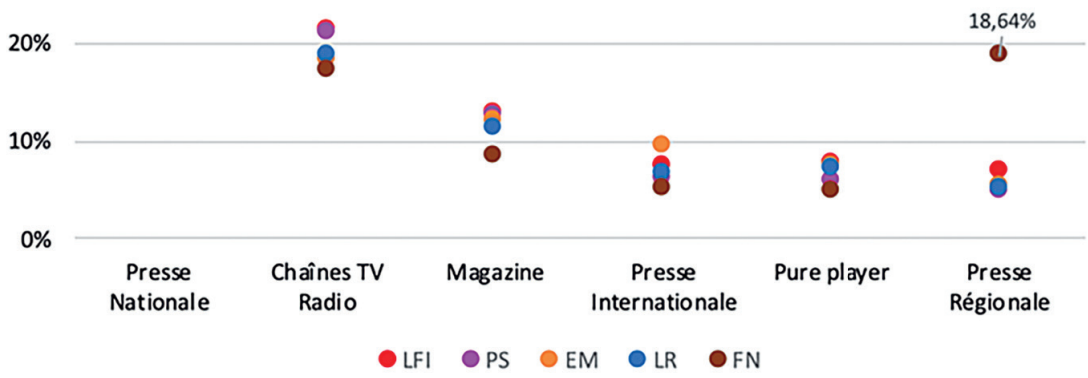

Figure 3. Part des types de presse dans les publications Facebook

Analyse lexicale des contenus textuels introduisant les articles de la PQR

Après avoir analysé la place de la $P Q R$ dans les pratiques d'infomédiation des différentes communautés politiques en nous basant sur une analyse quantitative du nombre d'articles partagés depuis les différents titres de la PQR de notre corpus, il est maintenant intéressant d'examiner en détail le type de nouvelles relayées par les groupes politiques et les utilisations qui en sont faites.

Pour cela, nous avons réalisé un examen qualitatif de l'ensemble des articles diffusés par les communautés politiques. Nous pouvons ainsi identifier cinq types d'informations différentes partagées depuis des titres de la $P Q R$ : - de l'information de service : date des réunions publiques, des déplacements de candidats;

- de l'information de proximité : ralliement ou déclaration d'un élu local, compte rendu de meeting;

- du moissonnage d’informations locales pour un objectif national : récolte des irrégularités dans les bureaux de vote au premier tour de l'élection; 
- de l'utilisation d'informations locales pour les réinterpréter dans la vision politique et l'agenda idéologique du groupe;

- de l'information nationale (sondage, ITV, déclaration, commentaires) que l'on pourrait trouver sur n'importe quel support.

Cet examen qualitatif démontre que toutes les communautés politiques relaient les deux premiers types (information de service et information de proximité), quoique dans une moindre mesure concernant le FN et LFI. Leur nombre moins important d'élus locaux est probablement un facteur explicatif de cette situation. Le cinquième type d'information se retrouve dans tous les groupes de façon marginale.

Pour aller plus loin, nous avons réalisé une analyse lexicale du contenu des messages diffusés par les administrateurs de communauté pour introduire les articles qu'ils ont partagés. La figure 4 montre par exemple comment un administrateur d'une page Facebook de LREM a partagé un article du journal Le Monde au sujet de l'accusation dont une eurodéputée du FN faisait l'objet. Dans le cas présent, le chapô introductif de l'article a été rédigé par des journalistes du Monde. Parfois, les administrateurs de communauté rédigent eux-mêmes un bref commentaire pour introduire le sujet de l'article qu'ils partagent depuis une source extérieure.

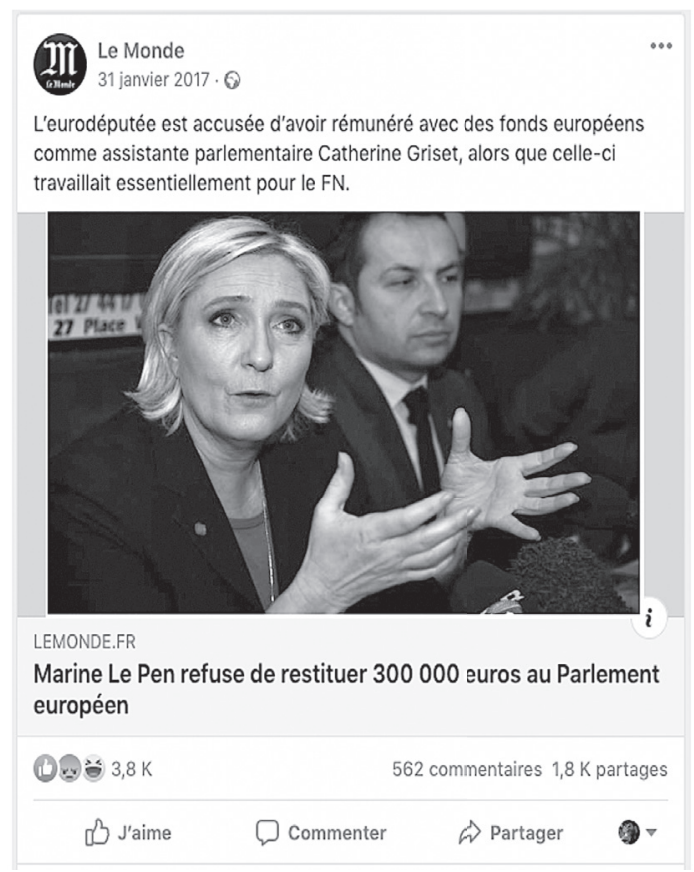

Figure 4. Exemple d'une publication Facebook partagée depuis Le Monde 
L'analyse statistique de ces messages permet d'identifier les sujets des articles que les administrateurs ont partagés depuis les sites internet de la $P Q R$. La figure 5 représente le dendrogramme qui synthétise l'analyse lexicale des publications Facebook contenant un lien vers un titre de la PQR. Cette analyse a été réalisée à l'aide du logiciel Iramuteq (voir supra). Elle souligne les types d'informations mobilisées par les différentes communautés politiques : - Les classes 6 et 7 renvoient de façon directe aux différents faits divers locaux qui constituent l'un des piliers du modèle éditorial actuel de la PQR;

- Les classes 1 et 9 sont constituées par des informations liées à des débats et des événements au sein de la gauche;

- Les classes 2 et 3 regroupent des articles évoquant la place de l'agriculture dans la politique européenne ;

- Et enfin les classes 8, 4 et 5 sont le fait d'articles consacrés à des constats d'irrégularités dans des bureaux de vote à l'issue du premier tour.

Après avoir identifié les sujets les plus traités dans les messages, il est intéressant de caractériser les thèmes surreprésentés au sein d'une communauté politique. La figure 6 montre ainsi quel est le poids de chaque classe lexicale dans les publications Facebook, contenant un lien vers la PQR, diffusées par les différentes communautés politiques. Plusieurs constats apparaissent et notamment des différences très fortes entre les communautés politiques quant aux types d'informations mobilisés. Les classes 6 et 7 (renvoyant à des articles publiant des faits divers) sont nettement surreprésentées dans la communauté FN. Puis les classes 2 et surtout 3, évoquant la place de l'agriculture dans la politique européenne, sont majoritairement mobilisées par la communauté LR. La classe 1, qui renvoie aux débats suscités à gauche par la candidature d'Emmanuel Macron, est plus présente dans la communauté LREM. Et enfin les classes 8,4 et 5, relatives à la dénonciation d'irrégularités dans certains bureaux de vote, sont plus caractéristiques des échanges au sein de la communauté LFI durant l'entre-deux-tours.

L'analyse et la discussion de ces résultats nous apportent des éléments sur la place du modèle éditorial de la $P Q R$ dans l'écosystème informationnel français et sur la façon dont les informations peuvent être mobilisées par des groupements politiques pour leur donner un sens conforme à leurvision du monde. Un premier élément saillant de nos résultats : la place unique que tient la $P Q R$ pour la construction d’informations de proximité. Quelle que soit la communauté politique, les informations mobilisées relèvent de la proximité.

Pour la communauté LR, il s'agit d'articles décrivant la situation agricole particulière des différents territoires. Les articles sélectionnés et cités concernent différents types d'agriculteurs, dans différentes parties de la France. Ils décrivent leurs situations et souvent leurs problèmes en les rapportant à la mondialisation. L'électorat agricole étant traditionnellement lié à un vote conservateur, il n'est pas étonnant de retrouver la mobilisation de ce type 


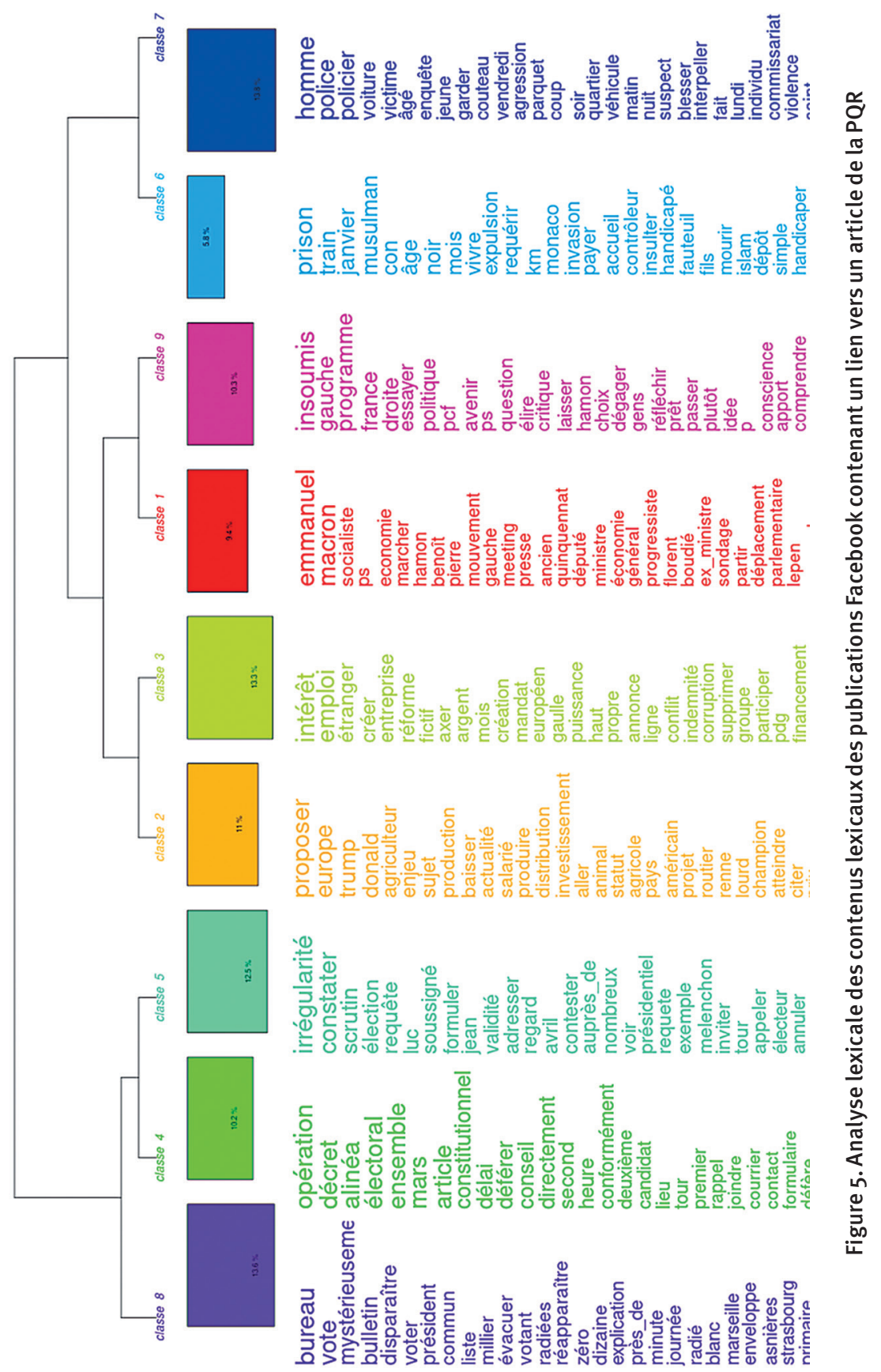




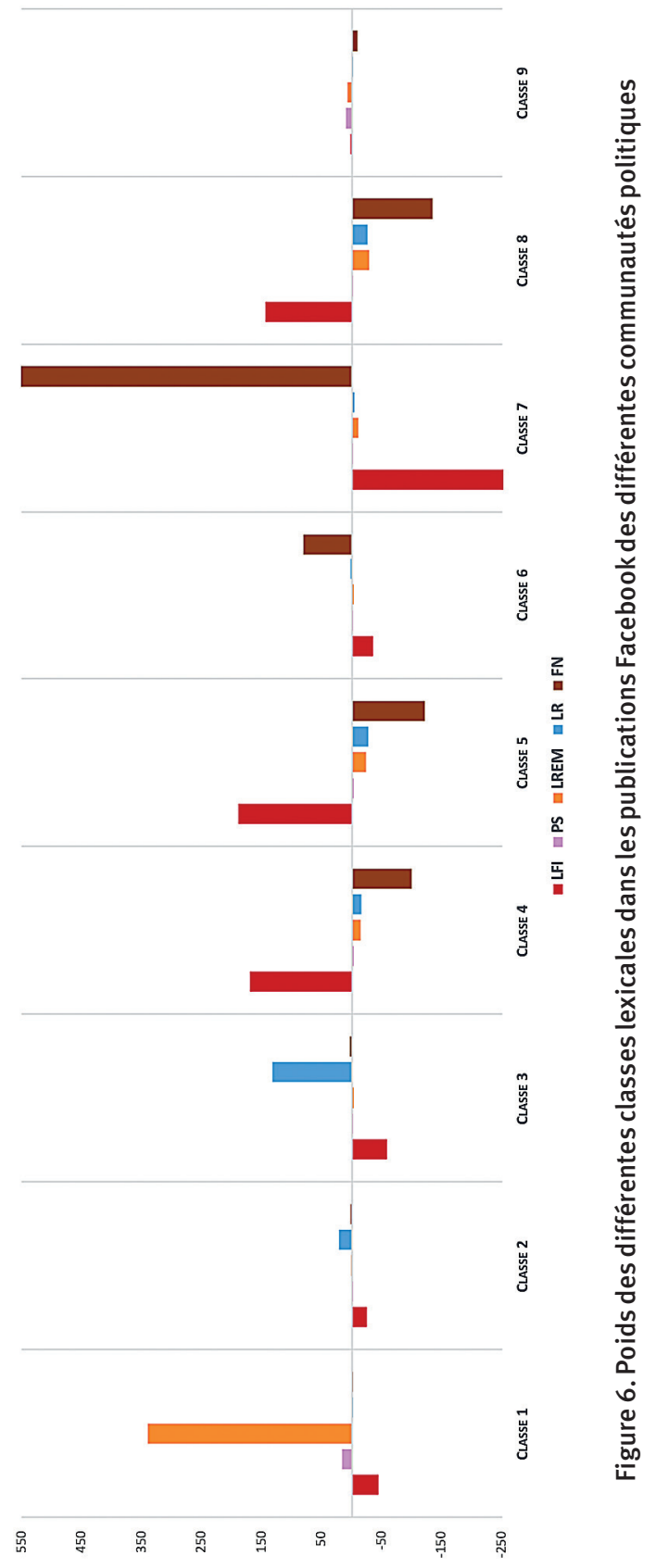


d'articles par la communauté LR. Cela correspond à la volonté d'activer un ressort électoral classique et de mettre en avant un thème sur lequel la droite est traditionnellement dominante. De même, la surreprésentation d'articles de la $P Q R$ relayés par cette communauté pour évoquer les problèmes liés à l'emploi ou à l'économie témoigne de sa volonté de les incarner dans des situations locales facilement identifiables par les lecteurs.

Pour LFI, le recours à la PQR est tardif et encore plus spécifique. Au lendemain du premier tour de l'élection présidentielle, la communauté LFI se mobilise pour en contester le résultat. Pour cela toutes les irrégularités constatées dans les bureaux de vote du pays sont recensées. La PQR est donc particulièrement mise à contribution, car elle sera le seul média à rendre compte de toutes les irrégularités constatées dans des bureaux de vote. Les entrefilets des correspondants sont ainsi systématiquement diffusés et mis ensemble par la communauté LFI.

Pour LREM, ce sont les articles de la classe 1 qui sont le plus mobilisés. Ils concernent majoritairement des informations sur les ralliements d'anciens membres du Parti socialiste, élus locaux, députés, ministres, à la candidature d'Emmanuel Macron. Ce mouvement que l'on a perçu nationalement à travers les prises de position de poids lourds politiques se retrouve à l'échelle locale avec des personnalités de moindre notoriété mais connues sur leur territoire. Là encore, il s'agit donc d'informations de proximité que seule la PQR est capable d'enregistrer dans toutes les parties du territoire.

Enfin et surtout viennent les articles des classes 6 et 7 qui ne concernent quasiment que la communauté FN. Il s'agit là de ce que l'on nomme des faits divers relatant agressions, vols, incivilités de tout ordre et leurs suites judiciaires, procès, jugements. Il ne s'agit pas d'affaires d'envergure ou de portée nationale mais de faits locaux que la $P Q R$, en lien avec les pouvoirs policiers et judiciaires, relaie systématiquement. Impossible de trouver des articles sur ces événements ailleurs que dans la $P Q R$, ils sont considérés comme l'une de ses spécialités et relèvent parfaitement de l'information de proximité.

Le premier constat des pratiques de médiation informationnelle des communautés politiques sur Facebook a donc trait aux particularités éditoriales de la PQR et aux informations qu'elle est la seule à donner. Des prises de position politiques d'élus locaux jusqu'aux recensions de problèmes dans des bureaux de vote en passant par des comptes rendus sur l'agriculture locale ou sur des vols, des agressions ou des incivilités, le maillage territorial de la $P Q R$ est unique. Ce type de presse possède donc une place à part dans le tissu informationnel français dont les pratiques de médiation informationnelle des communautés politiques sur Facebook témoignent. Ce constat, s'il n'est pas forcément contre-intuitif, est cependant très important quant à la place de la $P Q R$. À ce jour, et si l'on en croit les usages étudiés, aucun autre acteur informationnel n'a été capable de proposer une alternative à la PQR et à son réseau 
de correspondants, que l'on évalue à 36000 (Gimbert, 2012), soit autant que de communes françaises. Si aujourd'hui le modèle économique de ces titres est remis en cause (perte de lecteurs papier payants et de ressources publicitaires), ces particularités informationnelles faites de service et de proximité sont encore son apanage.

Cependant ce constat n'est pas le seul qui découle de l'analyse de nos résultats. Il s'avère que les faits désormais véhiculés par une $P Q R$ qui n'affiche pas une ligne éditoriale identifiable, ni idéologiquement ni dans la défense d'un point de vue sur le monde, donnent la possibilité d'une mise en cohérence autour d'interprétations politiques particulières. Pour le dire autrement : nous avons constaté une éditorialisation de la part des communautés politiques d'informations n'ayant a priori pas de sens dans leur contexte initial de production (selon la définition du fait divers par Roland Barthes, 1964), pour nourrir par l'exemple leur discours politique. Ainsi le local, dans sa singularité et à travers ses pratiques de médiation informationnelle, vient servir et ancrer un discours politique général.

En effet, si nous lisons les articles les plus partagés par les communautés et les commentaires qui les accompagnent sur la page Facebook ou sur le site de $P Q R$ qui les héberge, nous nous apercevons que toutes les communautés politiques procèdent ainsi : LR à travers l'accumulation de difficultés économiques localement situées dans les secteurs agricoles, commerciaux ou industriels, qui vient servir un discours contre les politiques du gouvernement et de l'Europe; LREM qui agrège des ralliements individuels pour apparaître comme un refuge pour les déçus du PS; LFI qui après l'élection utilise toutes les irrégularités constatées pour contester les résultats du premier tour.

\section{Les faits divers au cœur des pratiques de médiation informationnelle du FN}

Les analyses présentées dans les sections précédentes mettent en lumière des spécificités propres à la communauté du $\mathrm{FN}$ : ce sont les pages de ce groupe politique qui partagent le plus d'articles publiés par des titres de la $P Q R$, notamment ceux qui médiatisent des faits divers locaux à l'échelle d'une ville ou d'un quartier. Nous souhaitons donc maintenant faire un focus sur ces pages du FN pour mieux comprendre la manière dont elles convoquent et relaient des faits divers pour renforcer une interprétation du monde fondée sur l'insécurité, la délinquance, les incivilités et le rôle déterminant des populations étrangères comme cause de ces problèmes.

L'analyse du dendrogramme consacré spécifiquement aux messages (posts) de cette communauté montre effectivement que les classes 1, 7, 8, 6, 3 et 10 sont entièrement consacrées aux faits divers et à leurs conséquences judiciaires et policières, avec une place particulière réservée à des articles 


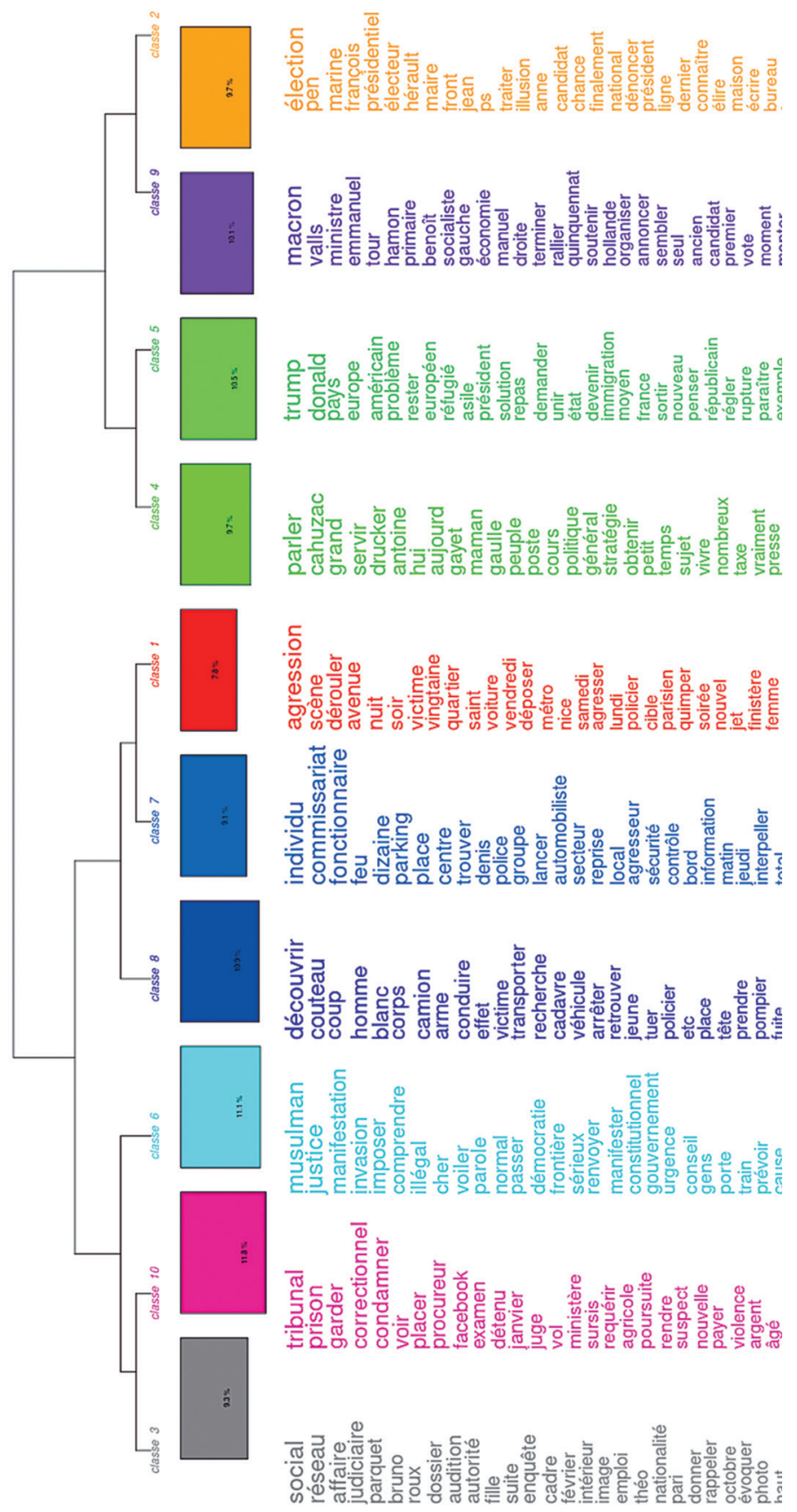


traitant de l'islam et de l'immigration, auxquels la classe 6 est presque entièrement consacrée (voir figure 7).

Quelques exemples d'articles relayés par le groupe FN nous permettent de comprendre pourquoi ils sont choisis et comment ils sont utilisés (voir tableau 2). Ces articles sont ceux qui ont été postés plusieurs fois (de 2 à 4 fois) dans des groupes liés au FN.

\begin{tabular}{l|l}
\hline Titre de l'article & Journal d'origine \\
\hline $\begin{array}{l}\text { Paris : des femmes victimes de harcèlement dans } \\
\text { les rues du quartier Chapelle-Pajol }\end{array}$ & $\begin{array}{l}\text { Le Parisien, 18/05/2017, } \\
18 \text { e arrondissement }\end{array}$ \\
\hline Vieux-Port : il fracture une quinzaine de bateaux & $\begin{array}{l}\text { La Provence, 20/05/2017, } \\
\text { édition Marseille }\end{array}$ \\
\hline $\begin{array}{l}\text { Une voiture fonce sur un policier à Béziers : } \\
\text { «On a tenté de le tuer» }\end{array}$ & $\begin{array}{l}\text { Midi Libre, 02/03/2017, } \\
\text { édition Béziers }\end{array}$ \\
\hline $\begin{array}{l}\text { Une «marche d'hommage» au cambrioleur tué } \\
\text { à Tourrettes-Sur-Loup organisée ce dimanche }\end{array}$ & $\begin{array}{l}\text { Nice Matin, 26/05/2017, } \\
\text { Cagnes }\end{array}$ \\
\hline $\begin{array}{l}\text { Un élève policier de Nîmes suspendu pour avoir } \\
\text { diffusé une vidéo de Daesh }\end{array}$ & $\begin{array}{l}\text { France 3 Occitanie, } \\
20 / 05 / 2017\end{array}$ \\
\hline $\begin{array}{l}\text { FOURMIES : Des pompiers agressés alors qu'ils } \\
\text { portent secours aux blessés }\end{array}$ & $\begin{array}{l}\text { La Voix du Nord, } \\
27 / 04 / 2017\end{array}$ \\
\hline Deux policiers blessés à l'arme blanche & $\begin{array}{l}\text { La Dépêche du Midi, 22/01/2017, } \\
\text { édition Lot-et-Garonne }\end{array}$ \\
\hline
\end{tabular}

Tableau 2. Exemples d'articles relayés par les pages Facebook du FN

Il n'est pas besoin de continuer cette liste qui comprend 539 articles. L'accumulation des faits divers locaux, épars dans les titres, suggère un climat de violence et d'insécurité. Les informations choisies s'intègrent parfaitement dans la vision du monde développée par le FN : dénonciation de violences faites aux femmes par des populations d'origine immigrée, présence des islamistes dans la société française, agression des forces de l'ordre, soutien à l'autodéfense. La présence des populations immigrées est ici assimilée à la violence envers les forces de l'ordre, aux cambriolages, à l'islam radical, à l'asservissement des femmes et au communautarisme. Tout cela s'opposant à la juste défense de leurs intérêts par les populations autochtones (comme cela apparaît explicitement dans l'interprétation qui est faite de l'article «Une "marche d'hommage" au cambrioleur tué à Tourrettes-sur-Loup organisée ce dimanche » publié dans Nice Matin). Nul besoin de rumeurs ou de fausses informations (Rebillard, 2017) pour construire une vision de la réalité et un agenda en opposition à ceux transmis par les médias nationaux. La recension des multiples petits faits allant tous dans le même sens permet de proposer une interprétation du monde social en accord avec l'idéologie politique du Front national. Seul et isolé, chacun des articles n'a un sens que local et parcellaire; mis ensemble et assortis d'un appareil de commentaires, tous ces articles construisent une 
vision du monde cohérente et parfaitement en accord avec l'idéologie politique du FN. Cette analyse sur l'utilisation des faits divers rejoint celle de Laurent Mucchielli sur l'insécurité et le sentiment d'insécurité dans le débat public médiatique comme «filon politique » exploité depuis toujours par l'extrême droite (Mucchielli, 2010), tout en étant spécifique, car résultant d'une éditorialisation qui échappe aux médias concernés.

Il convient de noter que les commentaires présents sous les articles en ligne sur les sites de presse, quand ils sont ouverts et peu modérés, peuvent être plus nombreux que sur la page Facebook. C'est le cas par exemple pour l'article du Midi Libre ou celui du Parisien, qui compte 124 commentaires pour le premier et 116 pour le second. Les commentaires postés sur ces sites sont tout à fait conformes à la tonalité du discours partisan de ce groupement politique. On peut faire l'hypothèse que le lien vers l'article sur la page Facebook incite la communauté à poster des commentaires sur le site de presse, mais nous n'avons pas d'informations suffisantes pour le confirmer.

L'utilisation politique des faits divers par le FN et ses supporters est en revanche un fait constaté par nos observations. La part importante de cette rubrique dans la $\mathrm{PQR}$ fait donc de ces titres des supports de communication et d'échanges d'idées privilégiés pour ce parti. Cette exploitation permet en outre de donner du corps à l'idéologie politique du FN et de l'incarner localement à travers des faits rapportés par la presse. C'est donc à un travail de politisation d'un certain type de nouvelles auquel on assiste, un travail de mise en cohérence idéologique d'informations éparses liées au modèle éditorial et économique de la $P Q R$. Les titres de $P Q R$ eux-mêmes n'éditorialisent pas ces informations en les rattachant à une vision du monde cohérente, mais l'accumulation de ces petites informations fait système grâce à la mise en lien effectuée par des groupes comme celui du FN sur Facebook.

En conclusion de ce travail, une question se pose quant à l'éditorialisation (et par conséquent quant à la politisation) de l'information locale (Rubado, Jennings, 2019). Les titres de la $P Q R$ ayant abandonné cette ambition depuis le milieu des années 1970 et la fin de la concurrence territoriale, ils se contentent désormais d'une cogestion du local avec les pouvoirs en place (Frisque, 2010). La mise en sens de l'ensemble de l'actualité locale n'est donc plus prise en charge par le média, et encore moins sous sa forme numérique. En effet, l'énonciation éditoriale du journal en ligne (Souchier, 1998) rend encore plus éparses les informations qui pouvaient avoir du sens à travers l'enchâssement territorial proposé par la version papier (Bousquet, 2016). Par conséquent, les groupes Facebook reprenant les informations et les intégrant dans la ligne politique ou éditoriale qu'ils défendent ont le champ libre pour leur donner le sens qu'ils souhaitent. 
Le rôle de la $\mathrm{PQR}$ consiste de plus en plus, semble-t-il, à récolter et à diffuser de l'information de proximité sans être en capacité d'en imposer sa propre éditorialisation. En effet, tant du point de vue des pratiques de médiation déléguées à des intermédiaires que des politiques de diffusion numérique qui conduisent à un éclatement de l'unité du journal, la cohérence éditoriale des titres de PQR tend à disparaître. Par conséquent, la mise en sens des informations produites doit désormais être recherchée du côté des intermédiaires de la médiation. La logique communautaire d'un réseau social numérique comme Facebook entraîne l'éparpillement et la multiplication de ces intermédiaires et rend nécessaires des observations multiples pour comprendre les logiques sociales désormais à l'œuvre dans la politisation du local.

\section{Références}

BARTHES Roland, 1964, "Structure du fait divers », dans Essais critiques, Paris, Seuil, p. $188-198$.

Berthaut Jérôme, Darras Éric, Laurens Sylvain, 2009, "Pourquoi les faits divers stigmatisent-ils? L’hypothèse de la discrimination indirecte », Réseaux, nº 157-158, p. 89-124.

Bougnoux Daniel, 2006, La crise de la représentation, Paris, La Découverte.

Boullier Dominique, 2014, «Médiologie des régimes d'attention», dans Y. Citton éd.,

L'économie de l'attention : nouvel horizon du capitalisme?, Paris, La Découverte, p. 84-108.

BOUSQUET Franck, 2016, «Les territoires de la presse locale et régionale», dans I. Dragan, N. Pelissier et D. Roventa Frumusani éd., Journalisme et transformations sociales : des anciens aux nouveaux médias, Paris, L'Harmattan, p. 39-60.

- 2015, «L'information service au cœur de la reconfiguration de la presse infranationale française », Réseaux, n¹93, p. 163-191.

CARDon Dominique, 2012, "Le parler privé-public des réseaux sociaux d'Internet», dans S. Proulx, M. Millette et L. Heaton éd., Médias sociaux : enjeux pour la communication, Québec, Presses de l’Université du Québec, p.33-45.

CHAdWICK Andrew, 2013, The Hybrid Media System: Politics and Power, New York, Oxford University Press.

FRANKLIN Robert, 2005, "McJournalism? The local press and the McDonaldization thesis », dans S. Allan éd., Journalism: Critical Issues, Maidenhead, Open University Press, p. 137-150.

FRISQUE Cégolène, 2010, «Une reconfiguration des espaces médiatiques et politiques locaux?», Revue française de science politique, vol. LX, nº 5, p. 951-973.

GIMBERT Christophe, 2012, "Le correspondant, un amateur de local engagé par son territoire », Sciences de la société, n 84-85, p. 51-65.

HAYES Danny, LAWLESS Jennifer, 2018, «The decline of local news and its effects: new evidence from longitudinal data », The Journal of Politics, vol. LXXX, n 1, p. 332-336. 
Institut Montaigne, 2019, Media Polarization "à la française"? Comparing the French and American Ecosystems, rapport de recherche, https://www.institutmontaigne. org/ressources/pdfs/publications/media-polarization-a-la-francaise-rapport.pdf (consulté le 5 mars 2020).

JenKInS Henry, 2008, Convergence Culture: Where Old and New Media Collide, New York, New York University Press.

JouËT Josiane, RIEFfEL Remy éd., 2013, S'informer à l'ère numérique, Rennes, Presses universitaires de Rennes.

LE CAROFF Coralie, 2018, «Le partage de l'actualité politique sur les profils personnels de Facebook», dans A. Mercier et N. Pignard-Cheynel éd., \#info : commenter et partager l'actualité sur Twitter et Facebook, Paris, Éditions de la Maison des sciences de l'homme, p. 199-225.

LE FLOCH Patrick, 1997, Économie de la presse quotidienne régionale : déterminants et conséquences de la concentration, Paris, L'Harmattan.

Marty Emmanuel, Rebillard Franck, Pouchot Stéphanie, LAfouge Thierry, 2012, «Diversité et concentration de l'information sur le web : une analyse à grande échelle des sites d'actualité français ", Réseaux, nº 176, p. 27-72.

Mercier Arnaud, Ouakrat Alan, Pignard-Cheynel Nathalie, 2018, «Facebook pour s'informer? Actualité et usages de la plateforme par les jeunes », dans A. Mercier et N. Pignard-Cheynel éd., \#info : commenter et partager l'actualité sur Twitter et Facebook, Paris, Éditions de la Maison des sciences de l'homme, p. 169-197.

MucCHIELLI Laurent, 2010, “"Insécurité”, "sentiment d'insécurité" : les deux veines d'un filon politique », Après-demain, n016, p. 3-6.

QuÉRÉ Louis, Dulong Renaud, 1978, Le journal et son territoire : presse régionale et conflits sociaux, Paris, Tours, EHESS, Université François Rabelais.

RATINAUd Pierre, 2014, «Iramuteq : Interface de R pour les Analyses Multidimensionnelles de Textes et de Questionnaires (Version o.7 alpha 2) [Windows, GNU/Linux, Mac OS X]», http://www.iramuteq.org (consulté le 5 mars 2020).

Rebillard Franck, 2017, «La rumeur du PizzaGate durant la présidentielle de 2016 aux États-Unis», Réseaux, n² 202-203, p. 273-310.

RebillaRd Franck, SMYRnAIos Nikos, 2010, «Les infomédiaires, au cœur de la filière de l'information en ligne : les cas de Google, wikio et paperblog », Réseaux, nº 160-161, p. 163-194.

REINERT Max, 1990, «ALCESTE, une méthodologie d'analyse des données textuelles et une application : Aurélia de Gérard de Nerval», Bulletin de méthodologie sociologique, $\mathrm{n}^{\circ} 26$, p. 24-54.

RIEDER Bernhard, 2013, "Studying Facebook via data extraction: the Netvizz application », dans WebSci'13: Proceedings of the 5 th Annual ACM Web Science Conference, p. 346-355.

RUBAdo Meghan, JenNings Jay, 2019, «Political consequences of the endangered local watchdog: newspaper decline and mayoral elections in the United States », Urban Affairs Review, https://doi.org/10.1177/1078087419838058 (consulté le 5 mars 2020).

Souchier Emmanuël, 1998, "L'image du texte pour une théorie de l'énonciation éditoriale », Les cahiers de médiologie, nº 6, p. 137-145. 


\section{Résumé / Abstract / Compendio}

\section{Pratiques de médiation informationnelle sur Facebook : l'appropriation politique de la presse quotidienne régionale lors de la campagne présidentielle de 2017}

Cet article étudie les pratiques de médiation informationnelle sur Facebook durant la campagne présidentielle française de 2017. Il se centre sur l'utilisation de la production de la presse quotidienne régionale $(P Q R)$ par les différentes communautés politiques. Il en ressort que les particularités éditoriales de ce type de presse sont exploitées par toutes les communautés mais que les sympathisants du Front national y ont davantage recours. Ils instrumentalisent le manque d'éditorialisation des faits divers produits par la $P Q R$ en les mobilisant pour servir leurs discours et leur idéologie politique.

Mots-clés : presse quotidienne régionale, médiation informationnelle, Facebook, faits divers, extrême droite

\section{Information-sharing practices on Facebook: the political appropriation of the daily press during the 2017 French presidential campaign}

This article examines information-sharing behaviour on Facebook during the 2017 French presidential campaign. It focuses on how the contents of the regional daily press (RDP) are used by the main political communities. We show that the editorial particularities of this local press are used by all communities, but above all by supporters of the "Front national" (national far-right party). They exploit the lack of editorialization of the short news items produced by the RDP to back up their rhetoric and promote their political ideology.

Keywords: regional daily press, information-sharing, Facebook, short news items, farright

\section{Prácticas de mediación informacional en Facebook: la apropriación política de la prensa cotidiana regional durante la campaña presidencial de 2017}

Este artículo estudia las prácticas de mediación informacional en Facebook durante la campaña presidencial francesa de 2017 . Se centra en el recurso a la producción de la prensa diaria regional ( $P Q R$ en francés) por las diferentes comunidades políticas. Se destaca de esta menera que las particularidades editoriales de este tipo de prensa son explotadas por todas las comunidades, pero que constituyen un mayor recurso para los simpatizantes del Front national. Instrumentalizan la falta de editoriales relativos a sucesos de la vida cotidiana producidos por la PQR mobilizándolos para servir sus discursos y su ideología política.

Palabras claves: prensa cotidiana regional, mediación informacional, Facebook, sucesos de la vida cotidiana, extrema derecha 